\title{
Email consultations in health care: 2-acceptability and safe application
}

\author{
Josip Car, Aziz Sheikh
}

Electronic communication promises to revolutionise the delivery of health care. In the second of two articles considering the potential for email consultations, Car and Sheikh summarise the evidence about public and professional attitudes to them and discuss how to ensure their safe use

Email may have an important role in augmenting and facilitating communication between patients and healthcare professionals. ${ }^{1}$ In this article we summarise the evidence describing public and professional attitudes to using email in routine clinical care and explore issues relating to ensuring such use is safe. Specifically, we aim to answer three questions:

- How acceptable is email consulting to members of the public and healthcare professionals?

- How can the quality and safety of email consultations be ensured?

- How can we safeguard patient information when using email for consulting?

\section{Attitudes towards email consultations \\ Public}

Recent research suggests that members of the public increasingly want personalised care delivered by a range of approaches, the exact means depending on individual preference and the nature of the problem. ${ }^{2}{ }^{\mathrm{w} 1}$ Several descriptive studies provide consistent evidence that people who use email would like email access to their doctors. ${ }^{3-7}{ }^{\text {w2-w8 }}$ For example, a recent US Harris poll revealed that $90 \%$ of survey respondents would welcome the opportunity to communicate with their doctors by email, with $56 \%$ of those surveyed stating that ability to communicate with their doctors electronically would influence their choice of doctor. Perhaps more surprising is that $37 \%$ indicated that they would be willing to pay for email access to their doctor. ${ }^{\mathrm{w} 9}$ This wish to communicate electronically seems to be driven by public perceptions that email communication offers the potential for quick and convenient access to healthcare advice that can supplement the traditional face to face consultation. ${ }^{3}$ w5 $\mathrm{w9}$

Between $1 \%$ and $10 \%$ of the US public communicate with their doctors electronically, most in only a limited capacity. ${ }^{78}$ w4 w6 ${ }^{\mathrm{w} 9}$ Electronic communications that the public would particularly like to see available include follow up emails after visits to doctors, receipt of personalised medical information, obtaining test reports, and submission of charts for monitoring chronic conditions such as diabetes and asthma. The public also thinks that doctors should use automated systems to help patients better manage preventive care (such as email reminders for impending flu vaccination). ${ }^{\text {w1 }}$ Many patients also wish to use email to book or cancel appointments, arrange non-urgent consultations, and request repeat prescriptions. ${ }^{5}$

Patients' satisfaction with email communication has been shown to be high, and this mode of communication is preferred over telephone calls by many for discussing non-urgent problems (see fig 1). However, satisfaction decreases sharply as response time increases, with patients expecting to receive a response within 48 hours of sending an email. ${ }^{5}{ }^{710}$ This is perhaps unsurprising because one of the main perceived benefits of email communication is its speed. ${ }^{\mathrm{w} 1}$

\section{Healthcare providers}

Most doctors in Western countries now have access to email at work (such as via the NHSNet in Britain). Surveys in the United States and Europe show that many doctors use email to communicate with patients (up to $20 \%$ in Europe and 25\% in the United States) but that most do so with only a small proportion of their patients $^{\mathrm{w} 9}{ }^{\mathrm{w} 11-\mathrm{w} 14}$ and generally only at the request of patients. ${ }^{\mathrm{w} 9}$ The main concerns of those who offer these services to patients are the protection of confidentiality, potential for errors and liability, and securing payment. ${ }^{6 \mathrm{w} 4 \mathrm{w11} \text { w15 } \mathrm{w} 16}$ Other concerns include identifying clinical situations where email communication is likely to be inefficient (perceived difficulties of responding to complicated messages, for example) and the challenges of incorporating email into existing work patterns without increasing overall workload and costs. $^{6 \text { w3 } 34 \text { w13 w17 }}$

Although doctors are selective in choosing which patients they communicate with by email, their selection criteria remain unclear. ${ }^{9}$ Qualitative studies of doctors communicating by email with their patients suggest that they view it as a useful addition to the communication options already available and believe that it offers the potential to enhance management of chronic diseases, facilitate patient education, improve continuity of care, and increase flexibility in responding to non-urgent issues. ${ }^{9 \text { w10 } 18 \text { w19 }}$ There is, however, little evidence to support these views. ${ }^{1}$

In 1999, 45 US specialty and state medical societies joined forces to create the Medem Network to connect doctors and patients online. ${ }^{\mathrm{w} 20}$ This online resource encourages patients to let their doctors know that they are interested in email access to care if such services are not already locally available. This mode of consulting has received little attention in UK plans for modernising information technology in the NHS., ${ }^{21}$

\section{Safe application of email consultations}

Wider adoption of email consultations seems to be hindered by concerns, voiced mainly by doctors, about the quality, security, and safety of email consultations. These concerns are receiving considerable attention as managed care organisations and insurance companies

Extra references (w1-w37) are listed on bmj.com
Department of Primary Care and Social Medicine, Imperial College London, London W6 8RP

Josip Car doctoral student in patient-doctor partnership

Division of

Community Health Sciences: GP Section, University of Edinburgh, Edinburgh

EH8 9DX

Aziz Sheikh professor of primary care research and development

Correspondence to: A Sheikh aziz.sheikh@ed.ac.uk 


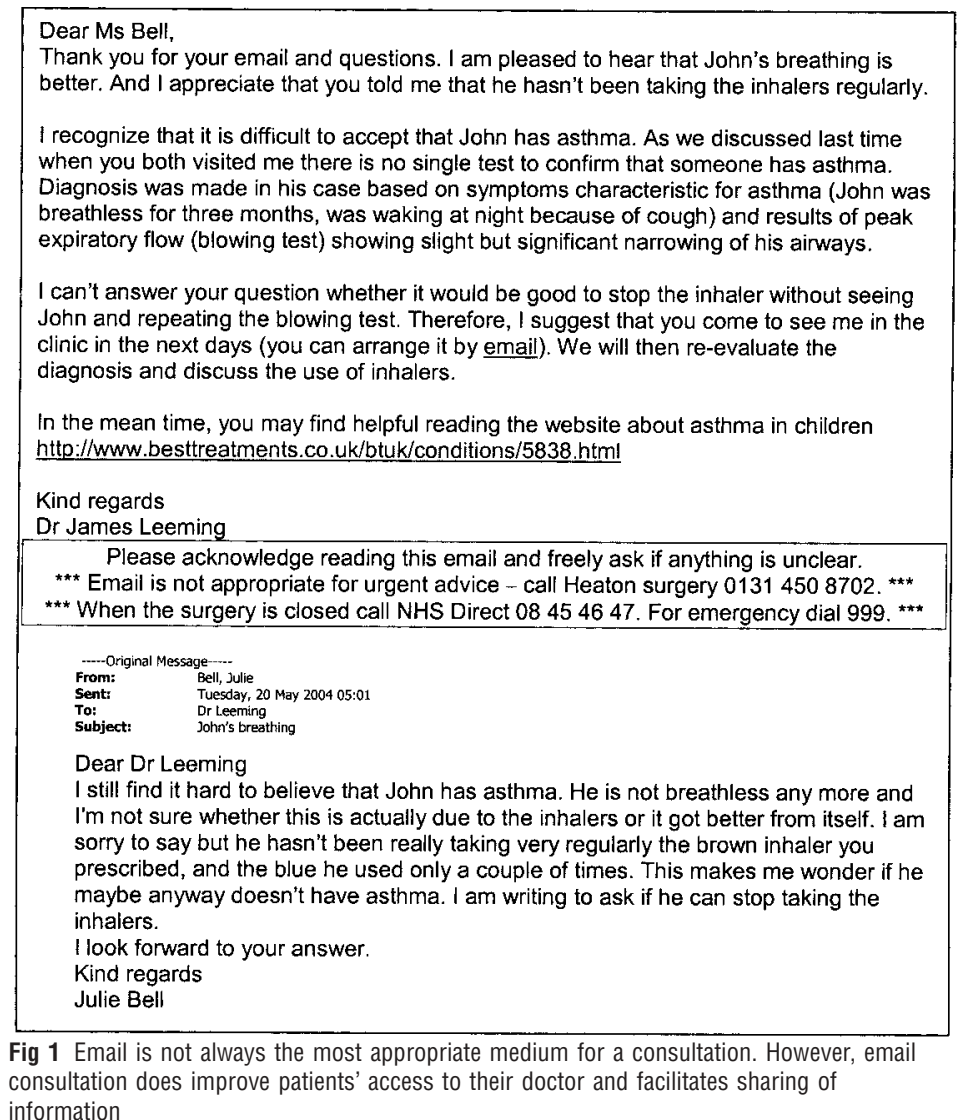

(mainly in the United States) strive to realise the potential that email consulting has to offer.

\section{Ensuring safety}

Email is generally viewed as a good means of communicating simple information and non-urgent requests between patients and doctors. In one study, however, up to $90 \%$ of patients who used email to communicate with their doctors relayed important and sensitive medical information electronically. ${ }^{3}$

In order to ensure safe and high quality email consultations, each specialty should develop policies and standards that will earn the trust of the public and healthcare professionals. ${ }^{10-13 \text { w22 w23 }}$ These should include guidance on organising the service, training, and strategies for mitigating risk during all stages of processing emails (receipt, triage, and response). ${ }^{11}$ Selection of optimal software for email consultation is extremely important (see box 1). ${ }^{\mathrm{w} 24}$

A key concern is whether email management is appropriate? Whenever in doubt doctors should revert to safer modes of consulting. A second concern is that the key points of a consultation have been correctly understood. Doctors can facilitate understanding by following the principles used in face to face or telephone encounters-such as the use of simple language, encouraging patients to ask questions, and summarising the main points covered. ${ }^{214}$ Additionally, a feedback loop may be used, whereby patients report what action they will take. If the subject being addressed is likely to require several emails back and forth, it is best to advise the patient to have a face to face or telephone consultation (see fig 1).
It may (initially) be appropriate to use a standard protocol clearly delineating the types of email communications that will be considered (for example, appointment scheduling, reporting of home records such as peak expiratory flow or blood pressure, ordering repeat prescriptions, obtaining test results, and consultations for a predefined set of conditions). Unsuitable topics, because of their complexity or sensitivity (because of the associated security concerns), may also be predefined. Patients should be advised not to use email for urgent communications. Similarly, when a doctor wants to ask a patient about symptoms that may require prompt action (such as chest pain or shortness of breath) a synchronous mode of consulting should be used. It may also be appropriate that each email includes a reminder about the importance of alternative forms of communication for emergencies.

Patients and doctors should communicate only through designated email addresses and services. Triage nurses may screen emails, as they do telephone calls, before they are routed to the appropriate person for a response. ${ }^{15}$ An automatic reply from the clinic can acknowledge receipt of a patient's email, and patients should be requested to acknowledge reading a doctor's email. Emails should be flagged as "unresolved" until an acknowledgment is received. Standardising specific communications (use of customised templates or protocols) to meet the needs of various specialties and tasks (such as repeat prescriptions) may make communication easier and increase quality and safety (see fig 2).

Box 1: Key features of optimal software for email consultations

- Ease of adoption (combining with existing technologies)

- Adaptability to an organisation's unique requirements for managing personal health information

- Seamless operating with existing infrastructures

- Enabling communication over various operating systems (such as Windows or Linux) and software programs

- User friendliness-easy to set up, manage, and use by doctors and patients

- Effective, invisible security over wired and wireless environments (without users needing to be aware of safeguards)

- Easy authentication methods

- Integration with existing medical records systems

- Possibility of the use of customised templates for email consultations

- Automation functions (such as automatic replies)

- System for preventing generation of messages to an addressee if previous messages remained unanswered for longer than set permissible time

- Integrated customisable message content filtering (if desired)

- Virus scanning

- Track and audit messaging system

- Archiving and logging

- Further considerations include which system is less error prone, needs less intensive support, and is more productive 


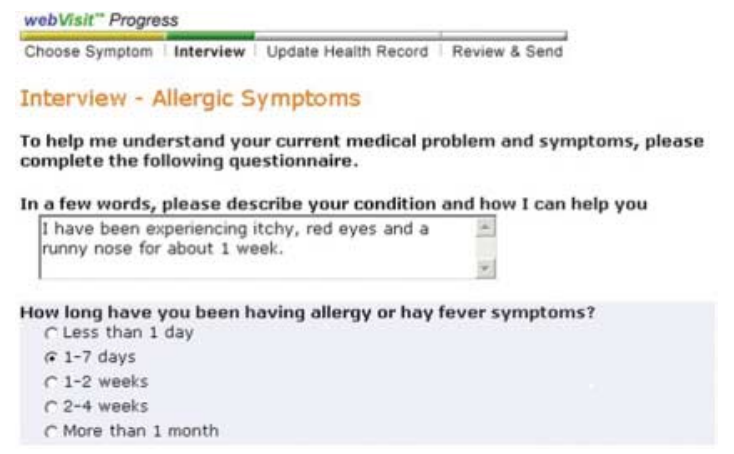

Fig 2 Example of a step in a structured, web based, email consultation for allergic symptoms. (Modified with permission from RelayHealth webVisit)

The written record of email consultations enables close monitoring and evaluation of appropriateness and safety. Whereas face to face and telephone consultations are rarely recorded verbatim (typically being documented with only a few key words), email provides direct evidence of patient-doctor conversation. Thus, email consultations have the potential to facilitate accurate record keeping. However, if the system is not seamlessly integrated with medical records, as is the case with many healthcare organisations, quality of recording may be poor. At present, many doctors need to "copy and paste" email messages into records or print out and file a paper copy of them. Collaboration between developers of secure email software and providers of electronic health records is needed to achieve their seamless integration.

Currently, email consultations with unknown patients are considered unsafe, and there are no agreed standards for such consultations (see box 2). A pragmatic approach to an unsolicited email from an

\section{Box 2: Email consultations with an unknown person}

Some healthcare professionals consult by email with patients they have never met in person..$^{16} 17$ w25 w26 For some patients, such anonymous email

communications may be less intimidating than face to face consultations. These communications may help them understand the nature of their problem and decide to seek help."27w*w0 However, the quality of such advice may vary greatly, and patients cannot be sure that replies are actually written by a doctor.

Currently, determining the identity and credibility of "cyberdocs" is difficult. In future, this could be overcome-for example, by using digital signatures (electronic proof of identity, which could also confirm that a doctor is officially licensed to provide such a service). Until standards are established, email consultations outside pre-existing patient-doctor relationships should not be considered as a method of rendering medical care..$^{1011} 1617$ w30-w32

Suggestion for an automatic reply to an unsolicited email

"Your email has not been read by any of the staff from Wood Thorpe Medical Centre. This is an automatic reply from a computer to an unsolicited email. No medical advice can be given without prior informed consent for email consultations. If you need any clarification about this automatic computer reply, please telephone 0171540 4987."

\section{Additional educational resources}

- Kane B, Sands DZ. Guidelines for the clinical use of electronic mail with patients. The AMIA Internet Working Group, Task Force on Guidelines for the Use of Clinic-Patient Electronic Mail. J Am Med Inform Assoc 1998;5:104-11.

- Medem. eRisk Working Group for Healthcare: guidelines for online communication. www.medem.com/phy/phy_eriskguidelines.cfm (accessed 7 May 2004)

unknown person or someone unregistered for use of email consultations is to reply with a standard disclaimer.

\section{Safeguarding patient information}

The ethical considerations, professional etiquette, and legal rules that guide traditional communication between healthcare professionals and patients are equally applicable to email consultations. ${ }^{10} 1819$ w33 Patients should be informed of the potential risks and benefits of email use, the ramifications, safeguards for privacy and confidentiality, and the practice or hospital policies on when and how to use email.

Ideally, informed consent should be obtained from patients before email communication is started. ${ }^{\text {w3 }}$ Patients should know who will process and have access to emails, including the times when the addressee is unavailable. They should be informed about the time in which email will be read and replied to-such as by the end of the next working day. An email should be forwarded (for example, to a specialist) or edited only with the sender's consent.

Email use in health care has developed without encryption. The security of unencrypted email is low, and email content can be inadvertently disclosed on the internet or local computer. Many countries now oblige healthcare organisations to follow the same strict data protection rules as do commercial institutions such as banks. As well as firewalls and conventional network security to protect content stored on an organisation's network, software that helps to achieve secure email communication (either web based or with standard email software) is available from several companies (such as Secure Data in Motion, Sigaba, ${ }^{\text {w35 }} \mathrm{Medem}^{\mathrm{w} 20}$ ). A critical factor in any solution for ensuring security is its user friendliness, and this may differ for patients and doctors and with different clinical settings and purposes. ${ }^{\text {w36 }}$ Safeguarding patient information also depends equally on paying due attention to organisational and technical considerations. Doctors risk breaching patient confidentiality if they use non-secure email with patients.

\section{Conclusions}

The Institute of Medicine considers "a sustained partnership between patients and clinicians" as a critical element of primary care. ${ }^{\text {w37 }}$ Email communication can help achieve this at a time when the personalised doctor-patient relationship is under threat from the increasing demands on health services.

Using email for patient-doctor communication increases patient choice in the way health care is received. To date, its use has largely been patient led, 


\section{Summary points}

National surveys show that patients increasingly want to be able to communicate with healthcare professionals by email

Few doctors currently do so: professional concerns centre on the quality of consultations, confidentiality, liability, and the challenge of recovering fees

Early email use in health care has grown without an adequate supporting infrastructure to address security issues

Ensuring privacy, confidentiality, and security of information is vital for email consultations, and various user friendly safeguards are now becoming available

Email consultations mark a radical shift from the traditional oral modes of communication: both patients and doctors need education in how to use them safely and effectively

with healthcare organisations slow to adopt it. ${ }^{20}$ Making email communication more readily accepted as a part of routine medical practice should be a key objective of the UK NHS information technology strategy. Widespread use in clinical practice requires the coordinated action of health professional organisations, patient representative groups, policy developers, and the information technology industry.

We thank Professors Azeem Majeed, George K Freeman, and Martyn R Partridge for their critical comments on an earlier draft of this manuscript.

Contributors: AS and JC conceived the idea for this review. JC conducted the searches, evaluated the study quality, and analysed the data. AS contributed to the search design, quality evaluation, and data analysis and interpretation. JC wrote the first draft of the paper; both authors jointly wrote the paper subsequently. Both authors are guarantors for the paper.
Funding: JC is supported by research awards from the Ministry of Education, Science and Sport, Slovenia, Ad Futura Foundation, and Universities UK (ORS award).

Competing interests: None declared.

1 Car J, Sheikh A. Email consultations in health care: 1-scope and effectiveness. BMJ 2004;329:435-8

2 Car J, Sheikh A. Telephone consultations. BMJ 2003;326:966-9.

3 Neill RA, Mainous AG III, Clark JR, Hagen MD. The utility of electronic mail as a medium for patient-physician communication. Arch Fam Med 1994;3:268-71.

4 Mandl KD, Kohane IS, Brandt AM. Electronic patient-physician communication: problems and promise. Ann Intern Med 1998;129:495-500.

5 Couchman GR, Forjuoh SN, Rascoe TG. E-mail communications in family practice: what do patients expect? J Fam Pract 2001;50:414-8.

6 Kleiner KD, Akers R, Burke BL, Werner EJ. Parent and physician attitudes regarding electronic communication in pediatric practices. Pediatrics 2002;109:740-4.

7 Sittig DF, King S, Hazlehurst BL. A survey of patient-provider e-mail communication: what do patients think? Int J Med Inf 2001:61:71-80.

8 Baker L, Wagner TH, Singer S, Bundorf MK. Use of the internet and e-mail for health care information: results from a national survey. JAMA 2003;289:2400-6

9 Patt MR, Houston TK, Jenckes MW, Sands DZ, Ford DE. Doctors who are using e-mail with their patients: a qualitative exploration. $J$ Med Internet Res 2003;5(2):e9.

10 Medem. eRisk Working Group for Healthcare: guidelines for online communication. www.medem.com/phy/phy_eriskguidelines.cfm (accessed 7 May 2004)

11 Kane B, Sands DZ. Guidelines for the clinical use of electronic mail with patients. The AMIA Internet Working Group, Task Force on Guidelines for the Use of Clinic-Patient Electronic Mail. J Am Med Inform Assoc 1998;5:104-11.

12 US Department of Health \& Human Services. Medical privacy-national standards to protect the privacy of personal health information. www.hhs.gov/ocr/hipaa/ (accessed 6. May 2004).

13 NHS Information Authority. NHSnet: safe computing guidance. www.nhsia.nhs.uk/nhsnet/pages/emailmessaging/policies/ safe_comp_guide.asp (accessed 6. May 2004).

14 Car J, Freeman GK, Partridge MR, Sheikh A. Improving quality and safety of telephone based delivery of care: teaching telephone consultation skills. Oual Saf Health Care 2004:13:2-3.

15 Katz SJ, Moyer CA, Cox DT, Stern DT. Effect of a triage-based e-mail system on clinic resource use and patient and physician satisfaction in primary care. a randomized controlled trial. J Gen Intern Med 2003;18: $736-44$.

16 Eysenbach G, Diepgen TL. Evaluation of cyberdocs. Lancet 1998; 352:1526.

17 Eysenbach G, Diepgen TL. Responses to unsolicited patient e-mail requests for medical advice on the world wide web. JAMA 1998; 280:1333-5.

18 Department of Health. Confidentiality:NHS Code of practice. London: $\mathrm{DoH}$ 2003. (www.dh.gov.uk/assetRoot/04/06/92/54/04069254.pdf, accessed 6 May 2004.)

19 Anderson R. Security in clinical information systems. London: BMA, 1996.

20 Committee on Ouality Health Care in America, Institute of Medicine. Crossing the quality chasm: a new health system for the 21st century. Washington, DC: National Academy Press, 2001.

\section{A lesson from across the Irish Sea}

Our annual Easter visit to see my mother in law provides an opportunity for some walking, time with the children, and, perhaps most importantly of all, grown up time in the numerous local pubs. At this year's visit not much had changed on the outside since last year-perhaps a few more shifty characters hanging around the pub doorways, but not so that you'd notice. Inside, everything also seemed much the same at first: the usual faces at the bar, the hum of conversation, the laughter, but was there something missing?

The pints took their usual few minutes to pour. (A perfectly presented pint of Guinness has always been an art form for Irish bar staff.) We sat down, took a deep breath in, and relaxed with those first sips of drink. Then it became apparent what was missing; the deep breath wasn't followed by the usual cough brought on by the smoke-filled atmosphere. The air was clear, we could see the other side of the pub with no haze to obscure our view. There were no ashtrays to be seen, and now we noticed the signs warning of financial penalties should anyone start smoking.
The ban on smoking had been introduced in Ireland just a few weeks before. At the time, I was sceptical as to how well this would be adhered to, as other licensing rules, such as closing time, often seemed to be neglected. But here was clear evidence that the law was being enforced.

The numbers of people in the pubs seemed the same, and neither were people heading outside every few minutes. Talking to the locals, we found that, although the law was unpopular, people had to admit that they now smoked fewer cigarettes. If there was a worry, it was the increase in bar snacks being eaten. Fingers that previously might have been occupied with cigarettes were now busy with crisps and nuts instead.

What a joy to come back from a night out without cigarette smoke clinging to every fibre and to be able to wear your clothes again the next day.

Andrew Thorns consultant and honorary senior lecturer in palliative care, Pilgrims Hospice and East Kent NHS Trust, Margate 